\title{
The Assignment of the Absolute Configuration of Non-Cyclic Sesquiterpenes by Vibrational and Electronic Circular Dichroism: The Example of Chiliadenus lopadusanus Metabolites
}

\author{
Giuseppe Mazzeo $^{1,+(\mathbb{D}}$, Alessio Cimmino ${ }^{2,+} \mathbb{D}$, Giovanna Longhi ${ }^{1}\left(\mathbb{D}\right.$, Marco Masi $^{2}(\mathbb{D})$, Antonio Evidente ${ }^{2}$ \\ and Sergio Abbate $1, *$ (D) \\ 1 Dipartimento di Medicina Molecolare e Traslazionale, Università degli Studi di Brescia, Viale Europa 11, \\ 25123 Brescia, Italy; giuseppe.mazzeo@unibs.it (G.M.); giovanna.longhi@unibs.it (G.L.) \\ 2 Dipartimento di Scienze Chimiche, Università di Napoli Federico II, Complesso Universitario Monte \\ Sant'Angelo, 80126 Napoli, Italy; alessio.cimmino@unina.it (A.C.); marco.masi@unina.it (M.M.); \\ antonio.evidente@unina.it (A.E.) \\ * Correspondence: sergio.abbate@unibs.it; Tel.: +39-030-3717415 \\ + These two authors equally contributed to this work.
}

Citation: Mazzeo, G.; Cimmino, A.; Longhi, G.; Masi, M.; Evidente, A.; Abbate, $\mathrm{S}$. The Assignment of the Absolute Configuration of Non-Cyclic Sesquiterpenes by Vibrational and Electronic Circular Dichroism: The Example of Chiliadenus lopadusanus Metabolites. Biomolecules 2021, 11, 1902. https:// doi.org/10.3390/biom11121902

Academic Editor: Vladimir N. Uversky

Received: 23 November 2021 Accepted: 16 December 2021 Published: 18 December 2021

Publisher's Note: MDPI stays neutral with regard to jurisdictional claims in published maps and institutional affiliations.

Copyright: (c) 2021 by the authors. Licensee MDPI, Basel, Switzerland. This article is an open access article distributed under the terms and conditions of the Creative Commons Attribution (CC BY) license (https:/ / creativecommons.org/licenses/by/ $4.0 /)$.

\begin{abstract}
Hydroxynerolidol, 9-oxonerolidol, and chiliadenol B are three farnesane-type sesquiterpenoids isolated from Chiliadenus lopadusanus that have shown an interesting activity against human pathogens as Gram+ and Gram - bacteria resistant to antibiotics. However, the absolute configuration (AC) of these interesting sesquiterpenes has not been assigned so far. Vibrational and electronic circular dichroism spectra have been recorded and correlations are pointed out for the three compounds. Density functional theory (DFT) calculations are used in conjunction with Mosher's method of investigation to assign AC. Statistical analysis is considered to quantitatively define the choice of AC from VCD spectra.
\end{abstract}

Keywords: Chiliadenus lopadusanus; farnesane-type sesquiterpenes; 9-hydroxynerolidol; 9-oxonerolidol and chiliadenol B; VCD; ECD; absolute configuration; DFT calculations

\section{Introduction}

The bioactive metabolites produced by plants, microorganisms, lichens, and algae had shown to possess a plethora of different biological activities such as phytotoxic, antibacterial, antiviral, anticancer, antitumor, algicide, antifungal, enzyme inhibiting, immunostimulant, antiplatelet aggregation, cytotoxic and antiplasmodial activities. They belong to all different classes of natural compounds as alkaloids, hydrocarbons, lipids, sterols, esters, fatty acids, derivatives of amino acids, terpenoids and aromatic compounds, etc., [1-4]. Among them, many could have potential practical application in agriculture as natural safe pesticides [5,6] and others could be the active principle of new drugs [7-9].

Natural products, in particular, those with a new carbon skeleton, could represent an efficient solution to discover and develop new drugs to overcome antimicrobial resistance and treat biofilm-related infections. This is a real emergency since in recent decades many pathogens causing human infection rapidly increased their antibiotic resistance reducing the efficacy of therapies. Recently the antibiotic activity of natural compounds, such as epiepoformin, sphaeropsidone, and sphaeropsidin A was tested against some species of Gram+ and Gram - bacteria which are considered common opportunistic pathogens inducing human heavy infections. Their combination increased their efficacy while sphaeropsidin A also inhibited biofilm formation. These results are very promising and suggested to some of us how to develop a suitable drug formulation, in particular, for wound protection against serious infection advancement [10].

These results prompted further research on fungi and plants to identify the bioactive natural substances with antibiotic activity against human pathogens as antibiotic-resistant 
bacteria. Thus, a screening of endemic plants collected in different regions of the Mediterranean basin was carried out to identify the best plant to reach this objective. The organic extract of $C$. lopadusanus, among all collected plants, showed high inhibition of some pathogenic bacteria growth. C. lopadusanus Brullo is an endemic plant growing spontaneously on Lampedusa Island, the largest Island of the Pelage archipelago, about $100 \mathrm{~km}$ from the North Africa coast and $200 \mathrm{~km}$ from Sicily coast [11].

The organic extract of $C$. lopadusanus showed very strong activity against both Gram+ and Gram - bacteria pathogens for the human species, which showed antibiotic resistance. This activity was essentially related to the presence of three farnesane-type sesquiterpenes, namely 9-hydroxynerolidol, 9-oxonerolidol, and chiliadenol B (1-3, Figure 1), respectively. The sesquiterpenes 1-3 were identified by comparing their optical rotation data (specific optical rotation: $[\alpha]^{25} \mathrm{D}=+5.2$ for $\mathbf{1},+14.4$ for 2 , and +4.0 for 3 ) and spectroscopic data (IR, UV, $1 \mathrm{D}^{1} \mathrm{H}$ and ${ }^{13} \mathrm{C}$ NMR and ESIMS) with the literature [12]. Their purity $>98 \%$ was ascertained by ${ }^{1} \mathrm{H}$ NMR, ESI MS, and HPLC.<smiles>[R]C(C=C(C)C)CC(C)=CCCC(C)(O)C=C</smiles>

1, $\mathrm{CL} 1,9-$-Hydroxynerolidol, $\mathrm{R}=\mathrm{H}$

4, R=S-MTPA

5, R=R-MTPA<smiles>C=CC(C)(O)CCC=C(C)CC(=O)C=C(C)C</smiles>

2, CL4, 9-Oxonerolidol<smiles>C=CC(C)(O)CCC=C(C)CC(=O)CC(C)C</smiles>

\section{3, CL5, Chiliadenol B}

Figure 1. Structures of 9-hydroxynerolidol, 9-oxonerolidol, and chiliadenol B (1-3).

9-Hydroxynerolidol and 9-oxonerolidol ( 1 and 2$)$ were isolated for the first time together from Solanum melongena [13]. The absolute configuration (AC) of 1 was tentatively assigned thereby reducing 9-oxonerolidol (2), and comparing its ${ }^{13} \mathrm{C} \mathrm{NMR}$ data with the natural one. According to Stoessl et al. [13], the product of the reduction was the expected mixture of two diastereomers, while the natural compound $\mathbf{1}$ appeared to be a mixture ( 4:1) of two diastereomers and both natural and synthetic diastereomeric mixtures of 1 have no optical activity. The AC of the bioactive form of sesquiterpene 2 was determined based on ${ }^{1} \mathrm{H}$ NMR and ORD data [13-15]. The AC of chiliadenol B (3) up to now has not been determined [16].

Considering these results that practically do not permit the unambiguous AC assignment of $\mathbf{1}$ and $\mathbf{2}$ and the still missing assignment of AC of $\mathbf{3}$ and considering strictly relationships between the AC and the biological activity of naturally occurring substances [17,18], we concluded that different methods, i.e., chiroptical ones, are needed to assign the AC of the three farnesane-type sesquiterpenes 1-3. Indeed, the latter papers demonstrate that the determination of $\mathrm{AC}$ is not a mere exercise to complete the knowledge about the chemistry of natural products but may tell us about their way of functioning as pharmaceutical agents.

This Manuscript describes the AC assignment of the three compounds, comprised of the configurations of the stereogenic carbon atoms C-9 for $\mathbf{1}$ and C-3 for all three sesquiterpenes, using the advanced Mosher method and extensive use of vibrational circular dichroism (VCD) spectroscopy. Indeed, while the former approach has met with numerous achievements and has been critically reviewed by Cimmino et al. [19], the latter technique has been increasingly used through the years [20,21]. For natural products, it has been used with success [22-25]. Even more relevant for the present work are the VCD 
and IR studies by Merten et al. [26,27] on linear diterpene natural products extracted from Bifurcaria Bifurcata algae. Herein we will make use of VCD and IR absorption data in the mid-IR region, and of IR spectra in the $\mathrm{CH}$ and $\mathrm{OH}$ stretching regions, the latter spectroscopic data still being relevant in the discrimination of diastereomers, albeit they not being chiroptical data. VCD in the $\mathrm{CH}$ and $\mathrm{OH}$-stretching regions will be cursorily considered (signals there are not fully reliable) and some limited use will be made of the corresponding IR spectra. Furthermore, following the recommendation of Polavarapu et al. [28], we will consider electronic circular dichroism (ECD) data in conjunction with VCD: we think that the synergistic use of the two types of data is particularly opportune in the present case since the expected number of possible conformers makes this study difficult, already noticed by Merten et al., for some diterpenes, namely elegandiol and other linear diterpenes from Bifurcaria family [26,27].

\section{Materials and Methods}

\subsection{General Experimental Procedures}

Optical rotation was measured in $\mathrm{CHCl}_{3}$ solution by a P-1010 digital polarimeter (Jasco, Tokyo, Japan). ${ }^{1} \mathrm{H}$ and ${ }^{13} \mathrm{C}$ NMR spectra were recorded at 400 and $100 \mathrm{MHz}$ in $\mathrm{CDCl}_{3}$ by Bruker spectrometers (Karlsruhe, Germany). ESIMS were recorded using LC/MS ESIMS-TOF system (Agilent 6230B, HPLC 1260 Infinity) (Milan, Italy). The HPLC separation was performed using a Phenomenex LUNA (C18 (2) $5 \mathrm{u} 150 \mathrm{~mm} \times 4.6 \mathrm{~mm}$ ) (Torrance, CA, USA). Analytical, preparative, and reverse-phase TLCs were carried out on silica gel (Merck, Kieselgel 60, F254, 0.25, 0.5 mm, and RP-18 F254s respectively) plates (Merck, Darmstadt, Germany). The spots were visualized by exposure to UV radiation, or by spraying first with $10 \% \mathrm{H}_{2} \mathrm{SO}_{4}$ in $\mathrm{MeOH}$, and then with $5 \%$ phosphomolybdic acid in $\mathrm{EtOH}$, followed by heating at $110^{\circ} \mathrm{C}$ for $10 \mathrm{~min}$. Column chromatography was performed using silica gel (Kieselgel 60, 0.063-0.200 mm) (Merck, Darmstadt, Germany).

\subsection{Plant Material}

Whole aerial parts of $C$. lapodusanus plants were collected fresh in Lampedusa Island (Italy) by Mr. Fabio Giovanetti and identified by Prof. G. Surico, University of Florence, Italy. The plant specimen is deposited in the collection of the Department of Plant Pathology, University of Florence. The air-dried sample was obtained as recently reported [10].

\subsection{Isolation of Fungal Metabolites and Synthesis of Ancillary Products}

Plant material $(450 \mathrm{~g})$ was extracted by $\mathrm{H}_{2} \mathrm{O} / \mathrm{MeOH}(1 / 1, v / v)$ and the organic extract was purified by a combination of column and TLC as recently reported [12] to obtain all samples as pure oils, namely for 9-hydroxynerolidol (1, $12.4 \mathrm{mg})$, 9-oxonerolidol (2, $11.7 \mathrm{mg})$ and chiliadenol B $(3,20.4 \mathrm{mg})$.

\subsubsection{Hemisynthesis of 4}

9-O-(S)- $\alpha$-Methoxy- $\alpha$-trifluoromethyl- $\alpha$-phenylacetate (MTPA) ester of $\mathbf{1}$ (4). To $\mathbf{1}(1.0 \mathrm{mg})$ in pyridine $(100 \mu \mathrm{L}),(\mathrm{R})-(-)-\mathrm{MPTA}-\mathrm{Cl}(10 \mu \mathrm{L})$ was added. The mixture was carried out for $4 \mathrm{~h}$ at $25^{\circ} \mathrm{C}$ and quenched by adding methanol and toluene. The mixture was evaporated using an $\mathrm{N}_{2}$ stream. The residue (1.3 mg) was purified by TLC, eluted with $\mathrm{CHCl}_{3} /$ isoPrOH $(95 / 5, v / v)$, yielding 4 as a homogeneous oil $(0.8 \mathrm{mg})$. It had: ${ }^{1} \mathrm{H}$ NMR, see Table 1 ; ESI MS (+) spectrum, $\mathrm{m} / \mathrm{z} 455[\mathrm{M}+\mathrm{H}]^{+}$. 
Table 1. ${ }^{1} \mathrm{H}$ NMR data of 9-O-(S)- and 9-O-(R)-MTPA of 9-hydroxynerolidol esters (4 and 5, respectively) ${ }^{1}$.

\begin{tabular}{ccc}
\hline & $\mathbf{4}$ & $\mathbf{5}$ \\
\hline position & $\delta_{\mathrm{H}}(\mathrm{J} \mathrm{in} \mathrm{Hz})$ & $\delta_{\mathrm{H}}(\mathrm{J} \mathrm{in} \mathrm{Hz})$ \\
1 & $5.2148 \mathrm{br} \mathrm{d}(17.5)$ & $5.2150(1 \mathrm{H}) \mathrm{br} \mathrm{d}(17.5)$ \\
2 & $5.0649 \mathrm{dd}(10.8,1.2)$ & $5.0658 \mathrm{dd}(10.7,1.1)$ \\
5 & $5.9134 \mathrm{dd}(17.7,10.8)$ & $5.9151 \mathrm{dd}(17.5,10.7)$ \\
6 & $2.0454 \mathrm{~m}(2 \mathrm{H})$ & $2.0466 \mathrm{~m}(2 \mathrm{H})$ \\
$\mathrm{H}-8 \mathrm{~A}$ & $5.1582 \mathrm{br} \mathrm{t}(6.9)$ & $5.1593 \mathrm{br} \mathrm{t}(6.7)$ \\
$\mathrm{Me}-12^{2}$ & $2.6624 \mathrm{~d}(5.5)$ & $2.6631 \mathrm{~d}(5.6)$ \\
$\mathrm{Me}-13^{2}$ & $1.3150 \mathrm{~s}$ & $1.3144 \mathrm{~s}$ \\
$\mathrm{Me}-14$ & $1.2822 \mathrm{~s}$ & $1.2821 \mathrm{~s}$ \\
$\mathrm{OMe}$ & $1.5778 \mathrm{~s}$ & $1.5798 \mathrm{~s}$ \\
Ph & $4.6763 \mathrm{~s}$ & $4.6766 \mathrm{~s}$ \\
\hline
\end{tabular}

${ }^{1}$ The chemical shifts are in $\delta$ values (ppm) from TMS. ${ }^{2}$ These two signals could be exchanged.

\subsubsection{Hemisynthesis of 5}

9-O-(R)- $\alpha$-Methoxy- $\alpha$-trifluoromethyl- $\alpha$-phenylacetate (MTPA) ester of $\mathbf{1}$ (5): To $\mathbf{1}(1.0 \mathrm{mg})$ in pyridine $(100 \mu \mathrm{L})$ was added $(\mathrm{S})-(+)-\mathrm{MPTA}-\mathrm{Cl}(10 \mu \mathrm{L})$ and the reaction was performed as above reported. The crude residue $(1.2 \mathrm{mg})$ was purified by TLC eluted with $\mathrm{CHCl}_{3} / \mathrm{isoPrOH}$ $(95 / 5, v / v)$, affording 5 as a homogeneous oil $(0.7 \mathrm{mg}) .5$ had: ${ }^{1} \mathrm{H}$ NMR, see Table 1; ESI MS (+) spectrum, $\mathrm{m} / \mathrm{z} 455[\mathrm{M}+\mathrm{H}]^{+}$.

\section{4. $V C D$ and ECD Spectroscopies}

VCD experimental studies were conducted using a Jasco FVS 6000 FTIR instrument equipped with a ZnSe photo-elastic modulator (PEM), working at $50 \mathrm{kHz}$ modulation, placed past a wire grid linear polarizer and with lock-in amplifier after detection, with either an MCT or an InSb liquid- $\mathrm{N}_{2}$ cooling device for the regions $850-1800 \mathrm{~cm}^{-1}$ and $2500-4000 \mathrm{~cm}^{-1}$ respectively. For the latter region, we report only the IR spectra, since experimental VCD spectra did not exhibit a good enough signal-to-noise ratio. Samples were dissolved in $\mathrm{CCl}_{4}$ with the following concentrations: $0.02 \mathrm{M}$ in $5 \mathrm{~mm}$ Infrasil quartz cuvette for the $\mathrm{OH}$-stretching region, $0.05 \mathrm{M}$ in $1 \mathrm{~mm}$ Infrasil quartz cuvette for the $\mathrm{CH}$ stretching region, and $0.25 \mathrm{M}$ in $200 \mu \mathrm{m} \mathrm{BaF}_{2}$ cell for the mid-IR region. 6000 scans were acquired for each case, and similar spectra were taken for the solvent and subtracted out. ECD experimental studies were conducted with the use of a Jasco 815SE instrument with samples dissolved in acetonitrile at $0.001 \mathrm{M}$ concentration in $1 \mathrm{~mm}$ quartz cuvettes. 5 scans per spectra were acquired using $1 \mathrm{~mm}$ Suprasil quartz cuvettes. ECD spectra of the solvent were recorded in the same conditions and subtracted thereafter from the sample ECD spectra. UV spectra were obtained from the same apparatus, from voltage-adjusted DC signals.

\subsection{Calculations: From $M M$ to DFT}

The Gaussian16 package [29] was extensively used to theoretically investigate compounds $\mathbf{1}$, and, to a lesser extent, $\mathbf{2}$. We decided to work for $\mathbf{1}$ with the $(3 S, 9 S)$ and $(3 R, 9 S)$ stereoisomers. We first used the MM routine to retain all conformers within the energy interval 0-5 kcal $/ \mathrm{mol}$; we then undertook the quantum DFT method to define conformers within 0-2 kcal/mol (B3LYP/6-311++G(2d,p) level of theory). Finally, for the defined conformers we calculated VCD spectra using the Stephens theory [30] embedded in Gaussian16 for the calculation of rotational strengths, assigning Lorentzian band-shapes to each fundamental transition $\left(10 \mathrm{~cm}^{-1}\right.$ bandwidth) and averaging them with Boltzmann population factors based on $\Delta \mathrm{G}$. No anharmonic correction was tried, as illustrated by us earlier on simpler cases [31,32]. Also due to missing treatment of anharmonicity, we do not report the comparison of computed and calculated spectra for the $\mathrm{CH} / \mathrm{OH}$ stretching regions, which notoriously are most affected by that phenomenon [31,32]. VCD spectra for the $(3 R, 9 R)$ and $(3 S, 9 R)$ stereoisomers were generated by simply reversing the signs 
of spectra calculated for the $(3 S, 9 S)$ and $(3 R, 9 S)$ stereoisomers. The two sets of IR spectra are coincident with the original $(3 S, 9 S)$ and $(3 R, 9 S)$ choices. Other levels of theory, namely B3LYP/TZVP, were tried but found unsatisfactory. ECD spectra were calculated for the very same conformers using the TD/CAM-B3LYP functional and 60 states; Gaussian band shapes were employed with $0.2 \mathrm{eV}$ bandwidth.

\section{Results and Discussion}

The organic extract of dried leaves of $C$. lopadusanus was purified as reported in the Materials and Methods section to afford the three farnesane type 9-hydroxynerolidol, 9oxonerolidol, and chiliadenol B (1-3) as pure oils. Their purity $>98 \%$ was ascertained by ${ }^{1} \mathrm{H}$ NMR, ESIMS, and HPLC analyses.

Preliminarily, the relative stereochemistry of 1-3 and, in particular, that of a double bond between C- 6 and C-7 was assigned by NOESY experiments. In the NOESY spectrum of 1, 2, and 3 the lack of correlation between Me-14 with H-6 in all compounds confirmed the E configuration assigned in literature $[13,19]$ to the double bond between C- 6 and C-7.

At that point, the AC at C-9 of $\mathbf{1}$ and C-3 of all the three compounds remains to be determined.

A first assay to determine AC at C-9 of $\mathbf{1}$ consisted in the application of the advanced Mosher's method. Sesquiterpene 1 was reacted with (R)-(-)- and (S)-(+)-MTPACl and yielded the corresponding monoesters 4 and 5 and their ${ }^{1} \mathrm{H}$ NMR spectra were carefully recorded and the values for the chemical shifts are reported in Table 1. Subtracting the chemical shifts of 5 from those of 4 (Table 1), the $\Delta \delta(\mathbf{4}-\mathbf{5})$ values for almost all the protons were determined and described pictorially in Figure 2.

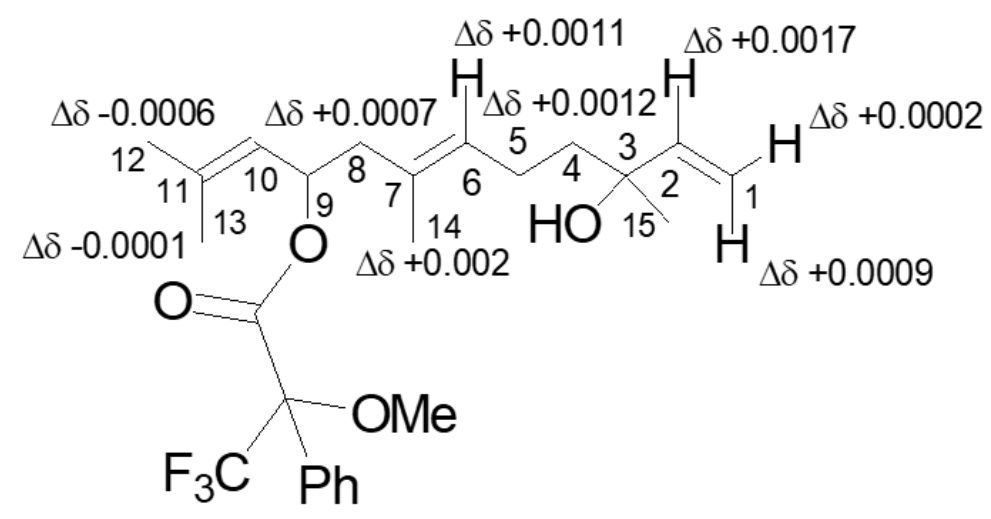

Figure 2. Structures of 9-O-S- and 9-O-R-MTPA esters of 9-hydroxynerolidol esters (4 and 5, respectively), reporting the $\Delta \delta$ value of each proton system.

Applying model A as reported in Cimmino et al. [19] the (S) configuration was assigned at C-9.

In Figure 3 we report the experimental IR and VCD spectra of the three compounds and in Figure 4 the corresponding UV and ECD spectra. We first seek common features in the spectra of the three molecules, namely we adopt an empirical correlative approach, and then we move to the comparison of experimental with DFT calculated spectra. We do this, since, due to the high number of conformers (vide infra), the exact determination of AC may be out of reach by the standard method. A few facts are worth pointing out from IR and VCD in the mid-IR region:

(i) At low wavenumbers, a feature at $921 \mathrm{~cm}^{-1}$ stands out, which exhibits negative VCD in all three cases and strong IR absorption. We believe this feature to be important for $\mathrm{AC}$ determination of stereo-carbon 3, common to all three molecules, possessing the same configuration there; 
(ii) at 1383 and $1450 \mathrm{~cm}^{-1}$ there is an IR doublet for all three compounds, which had been noticed also in refs [25,26]; no large VCD signal corresponds to these characteristic IR features;

(iii) the VCD spectrum of $\mathbf{1}$ is generally more intense than those for $\mathbf{2}$ and $\mathbf{3}$ and has some similarities with three out of four linear diterpenes' VCD spectra by Merten et al. [26,27], the most notable being the strong positive VCD band at ca. $1030 \mathrm{~cm}^{-1}$;

(iv) in the $C=C / C=O$ stretching regions a weak IR triplet is recorded for $1,(1630,1683$, and $1716 \mathrm{~cm}^{-1}$ ) much in the same way as observed by Merten et al. [26,27]. The three features are due to $C=C$ stretchings, which are known to exhibit weak absorption [33]. Interestingly with just one $\mathrm{C}=\mathrm{O}$ in both compounds, the spectral behavior in 2 and 3 is different: in 2 two strong bands appear at ca. 1630 and $1683 \mathrm{~cm}^{-1}$, due to the coupled $\mathrm{C}=\mathrm{C} / \mathrm{C}=\mathrm{O}$ stretchings; in 3 the single strong band at $1716 \mathrm{~cm}^{-1}$ is visible and is due to the isolated $\mathrm{C}=\mathrm{O}$ stretching, far away from all $\mathrm{C}=\mathrm{C}$ moieties.

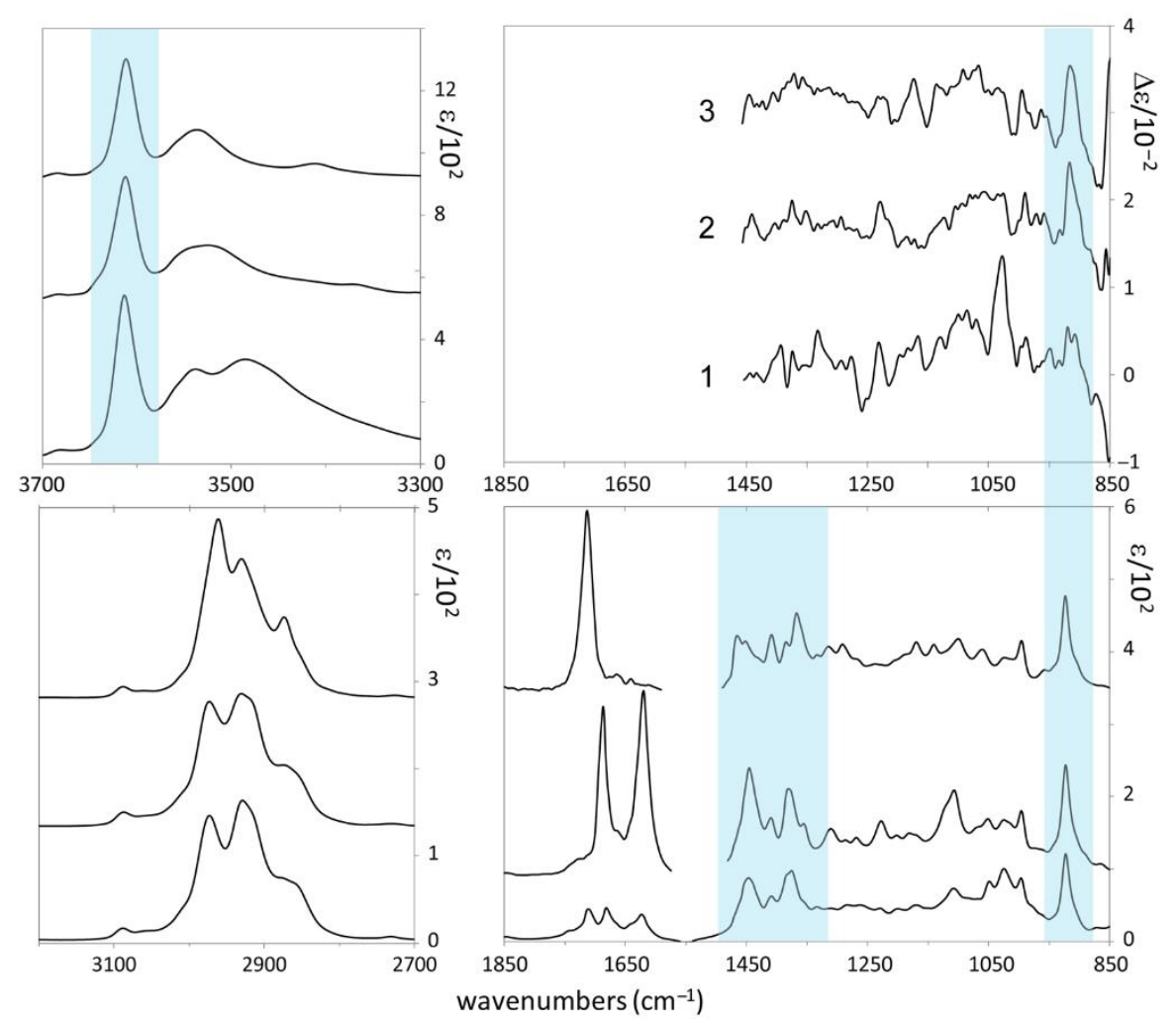

Figure 3. Right: Experimental VCD (top) and IR absorption (lower) spectra of 9-hydroxynerolidol, $\mathrm{R}=\mathrm{H}$ (1), of 9-oxonerolidol (2), and chiliadenol (3) in the mid-IR. Left: Experimental IR absorption spectra of 9-hydroxynerolidol, $\mathrm{R}=\mathrm{H}$ (1), of 9-oxonerolidol (2), and chiliadenol (3) in the $\mathrm{OH}$-stretching (top) and $\mathrm{CH}$-stretching (lower) regions. Features common to the three molecules are evidenced.

In the higher wavenumber $\mathrm{CH}$ - and $\mathrm{OH}$-stretching regions we point out the overall similarity in the three compounds of the $\mathrm{CH}$ stretching IR absorption spectrum. Several VCD spectra of cyclic terpenes [34-36] were recorded and presented in the past in the $\mathrm{CH}$-stretching region, with similar IR spectra as found here: we mention them here for sake of completeness, but we will not discuss them, for reasons reported in the Materials and Methods section. The IR absorption spectra in the $\mathrm{OH}$-stretching region looks more interesting since it exhibits a sharp feature at $3612 \mathrm{~cm}^{-1}$, common to all three compounds, and a broad feature at $3542 \mathrm{~cm}^{-1}$ in all three compounds, plus another broad hump centered at $3487 \mathrm{~cm}^{-1}$ for $\mathbf{1}$. Following Paoloni et al. [32] (see also the more recent work by Hartwig and Suhm [37]), who carefully investigated the cases of diols, we think that the $3612 \mathrm{~cm}^{-1}$ is due to the $\mathrm{OH}$-stretching in either the 3- or 9- position acting as an acceptor of $\mathrm{H}$-bond and behaving almost as an $\mathrm{OH}$-stretching "free" from $\mathrm{H}$-bond. We believe the 
$3542 \mathrm{~cm}^{-1}$ feature instead to be due to $\mathrm{OH}$ stretching in donor intramolecular $\mathrm{H}$-bonding for the 3 position (common to 1, 2, and 3) and the $3487 \mathrm{~cm}^{-1}$ feature to be due to $\mathrm{OH}$ stretching in donor intramolecular H-bonding for the 9 position (present only in $\mathbf{1}$ ).

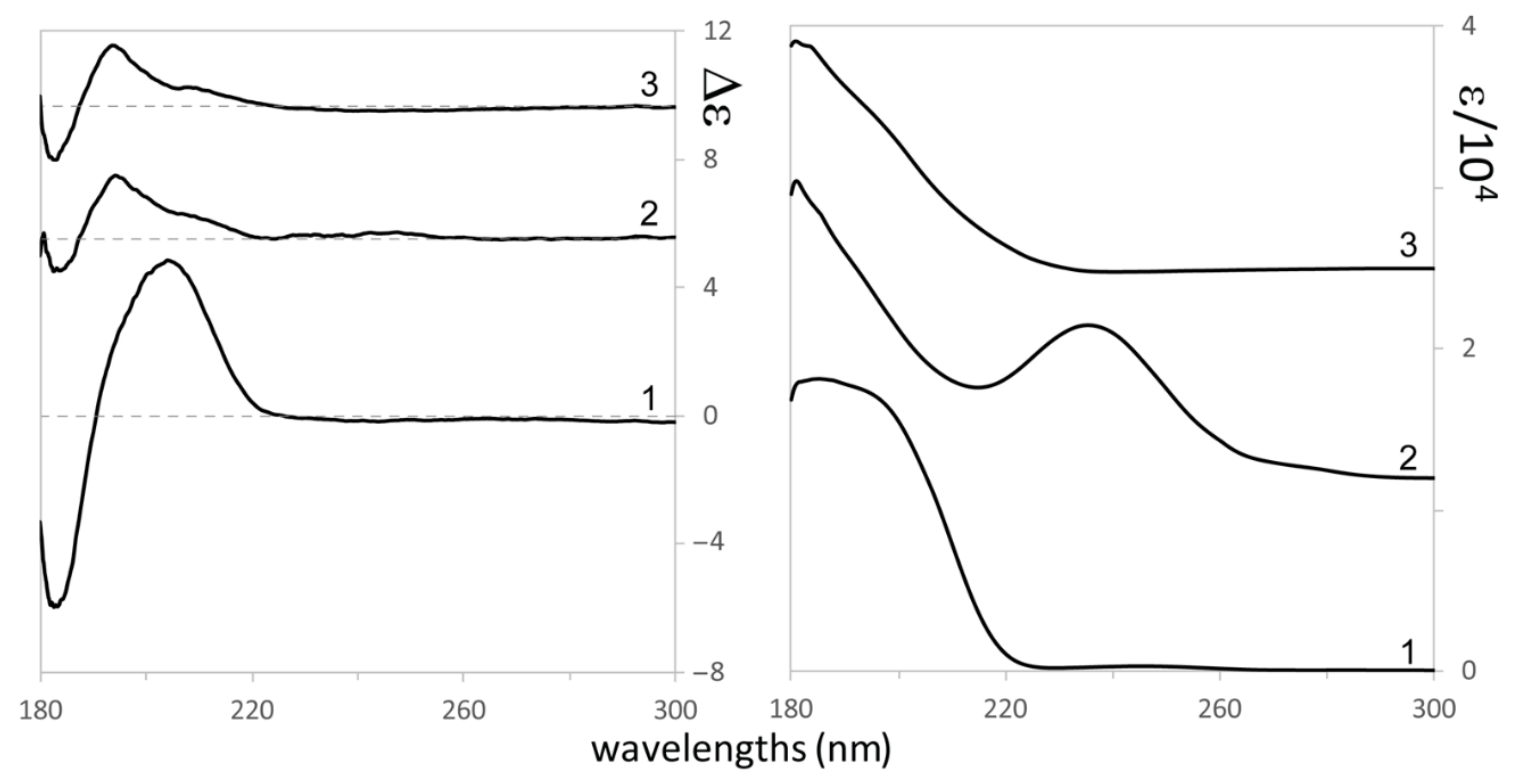

Figure 4. Experimental ECD (left) and UV absorption (right) spectra of 9-hydroxynerolidol (1), of 9-oxonerolidol (2), and chiliadenol (3).

Let us now come to consider Figure 4. A bisignate ECD feature extending from ca. 215 to $180 \mathrm{~nm}$ stands out in the spectrum and one might be tempted to correlate the three spectra. Yet some differences are noticed, which are important to consider: in $\mathbf{1}$ the intensity of the couplet is much more intense (from 5 to 10 times) than in 2 and $\mathbf{3}$, which are quite similar among themselves. Besides, the wavelengths of the observed features show differences, which are worth noticing: the positive feature in $\mathbf{1}$ is centered at $205 \mathrm{~nm}$, with a shoulder at $193 \mathrm{~nm}$; the negative feature is at $182 \mathrm{~nm}$. In 2 and 3 instead, while the negative component is at $182 \mathrm{~nm}$, the positive component is structured with a shoulder at $213 \mathrm{~nm}$ and the main positive band at $193 \mathrm{~nm}$. Earlier work on the diene chromophore [38,39] reviewed by Lightner and Gurst [40], address the possibility that the diene chromophore is distorted, so as to form a dissymmetric chromophore, or instead of being a symmetric chromophore dissymmetrically perturbed by the nearby oxygen atom. In the following, we will fully rely on TD-DFT calculations, which generally supersede previous interpretations [41]. Besides, the strong UV absorption at ca. $240 \mathrm{~nm}$ for 2, corresponding to a weak positive ECD effect, calls for a $n \rightarrow \pi^{*}$ transition, strongly coupled to diene transitions, in a way recalling what happens in the $1600-1700 \mathrm{~cm}^{-1}$ region of the IR absorption spectrum of 2 . The coupling may also justify enhanced UV absorption [40].

After the above empirical analysis of our chiroptical experiments for $\mathbf{1}$ (for which measured $\left.[\alpha]_{\mathrm{D}}^{25}=+4\right)$, for $2\left([\alpha]_{\mathrm{D}}^{25}=+14\right)$, and for $3\left([\alpha]_{\mathrm{D}}^{25}=+5\right)$, all OR measurements being carried out on chloroform diluted solutions, let us compare the DFT calculated IR and VCD spectra for 1 to corresponding experimental ones in the mid-IR region. Results are shown in Figure 5 for two choices of $\mathrm{AC}(3 S, 9 S)-\mathbf{1}$ and $(3 S, 9 R)-\mathbf{1}$. The calculated results for the other two possible configurations, namely $(3 R, 9 R)-\mathbf{1}$ and $(3 R, 9 S)-\mathbf{1}$, are not presented since their VCD can be obtained from those for $(3 S, 9 S)-\mathbf{1}$ and $(3 S, 9 R)-\mathbf{1}$ by simply reversing the signs, while their IR is the same as for (3S,9S)-1 and (3S,9R)-1. We limited calculations to $\mathbf{1}$, since the number of predicted conformers is huge, namely 15 for $(3 S, 9 S)-\mathbf{1}$ and 30 for $(3 S, 9 R)-\mathbf{1}$ (with ca. 400 conformers admitted by Molecular Mechanics): in the former cases, the predicted 15 conformers have $\Delta \mathrm{E}$ energies above the global minimum conformation $\leq 3 \mathrm{kcal} / \mathrm{mol}$, in the latter case the 30 predicted conformers have $\Delta \mathrm{E} \geq 2.5 \mathrm{kcal} / \mathrm{mol}$ above the global minimum. For reasons which will become clear 
in the subsequent discussion we averaged the calculated spectra for separate conformers through Boltzmann factors proportional to either $\mathrm{e}^{-(\Delta \mathrm{E} / \mathrm{RT})}$ or $\mathrm{e}^{-(\Delta \mathrm{G} / \mathrm{RT})}$ : results, though not drastically dissimilar, present some differences, which makes one choice preferable over the other. Upon inspection and based on IR spectra (IR is not a chiroptical technique, but is able to distinguish between diastereomers), we think that the best choice is (3S,9R) over $(3 S, 9 S)$; we also think that averaging over $\triangle \mathrm{E}$ provides better fitting than averaging over $\Delta \mathrm{G}$. Figure 3 shows that IR spectra in the $\mathrm{OH}$ stretching region indicate the presence of several intramolecular $\mathrm{HB}$, thus favoring compact conformers, as predicted for the most populated conformers according to the $\Delta \mathrm{E}$ statistics (vide infra). To a lesser extent than for IR, the same choice of AC and of average also holds for VCD.
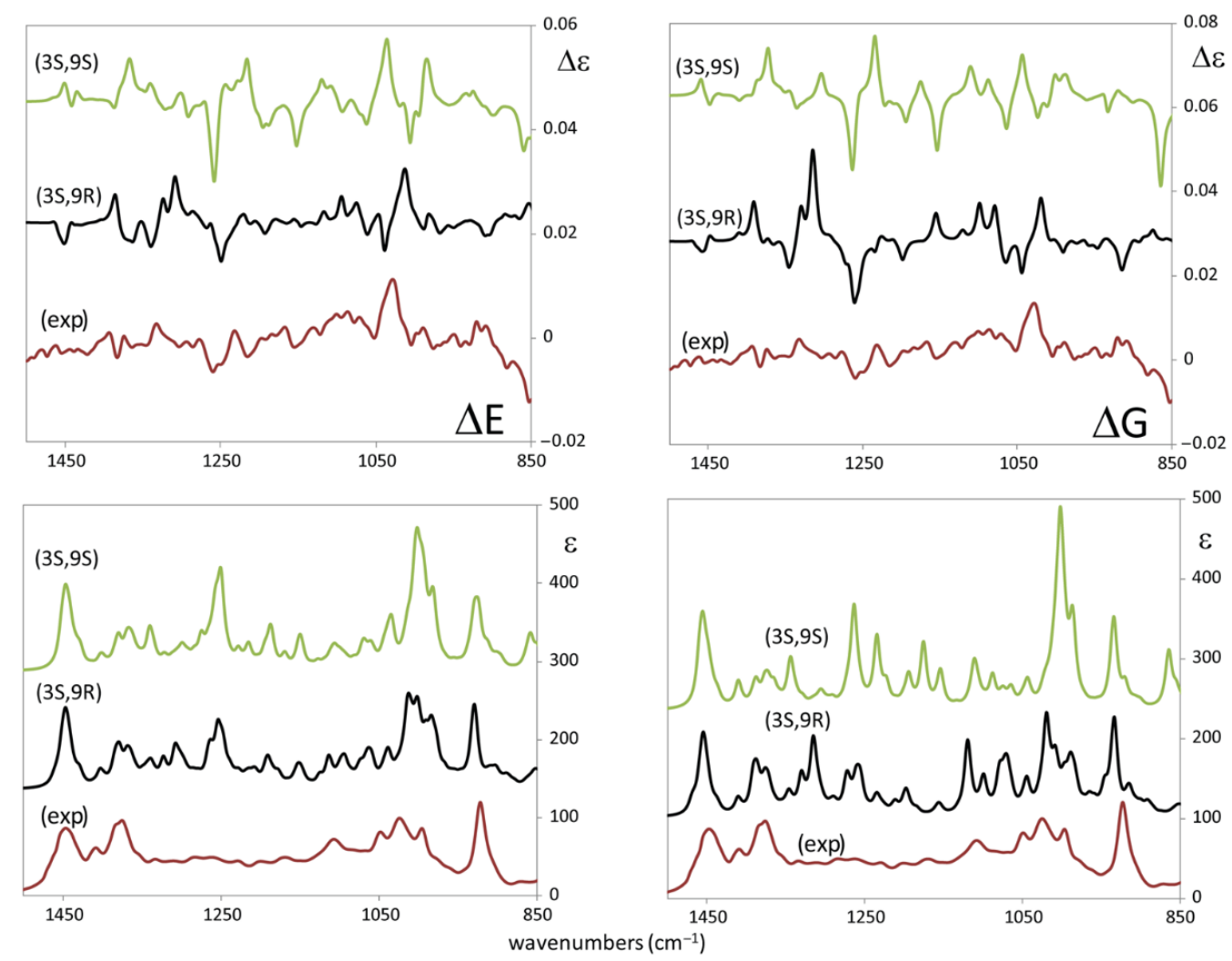

Figure 5. Comparison of experimental VCD (top) and IR absorption (bottom) spectra of 9hydroxynerolidol, $\mathrm{R}=\mathrm{H}(\mathbf{1})$, with corresponding DFT-calculated spectra of (3S,9R)-1 and (3S,9S)-1 with two different Boltzmann averages: on the left average carried out based on $\triangle \mathrm{E}$, on the right average carried out based on $\Delta \mathrm{G}$. Scaling factors 0.975 and 0.97 for $(3 S, 9 R)-\mathbf{1}$ and $(3 S, 9 S)-\mathbf{1}$ respectively. Scaling Factor for $(3 S, 9 S)=0.965$; SF for $(3 S, 9 R)=0.975$.

The choice made by visual inspection is quantitatively confirmed by calculating the similarity indices, as is commonly done in natural products chiroptical spectroscopy [42-46]. Indeed, as shown in the Supplementary Material (Figure S1 and Table S1) for the VCD spectra: for $(3 S, 9 R)$ we have 0.4 for parameter S.I. $(\Delta \mathrm{G})$ and $0.55(\Delta \mathrm{E})$, while for $(3 S, 9 S)$ we have 0.25 for parameter S.I. $(\Delta \mathrm{G})$ and $0.3(\Delta \mathrm{E})$. Similar conclusions are arrived at for parameter Sim_NN. In the Supplementary Material file, the results for other choices of the scaling factors around the values presented in the figures are explored, but the response is unambiguous, even though the differences between the parameters' values are not that big. (For the correct use of scaling factors, please consult NIST at: CCCBDB listing of precalculated vibrational scaling factors. Most importantly, based on this method, we also conclude that the $(3 R, 9 R)$ and $(3 R, 9 S)$ AC are to be excluded a fortiori; while we have to admit that the difference between the calculated indices for $(3 S, 9 S)$ and for $(3 S, 9 R) A C$ is 
not that great, in the remaining cases $(3 R, 9 R)$ and $(3 R, 9 S)$ we have even negative similarity SI indices, a safe argument not to accept the latter choices for AC.

Coming now to a more detailed comment on the results, we invite the interested reader to consult the Supplementary Material file, where the energetics of the conformers and the 3D-representation of the main conformers are given (Table S2 for the energetics in $\Delta \mathrm{E}$ and $\Delta \mathrm{G}$ for $\mathrm{AC}(3 S, 9 R)$ and Figures $\mathrm{S} 2-\mathrm{S} 4$ for the 3D-representation of $(3 S, 9 R)-$ conformers ordered in $\Delta \mathrm{E}$-population factors; Figures S5-S7 for 3D-representation of $(3 S, 9 R)$-conformers ordered in $\Delta \mathrm{G}$-population factors; Table $\mathrm{S} 3$ for the energetics in $\Delta \mathrm{E}$ and $\Delta \mathrm{G}$ for AC $(3 S, 9 S)$ and Figures S2-S4 for the 3D-representation of $(3 S, 9 S)$-conformers ordered in $\triangle \mathrm{E}$-population factors; Figures S5-S7 for 3D-representation of (3S,9S)-conformers ordered in $\Delta \mathrm{G}$-population factors). We observe that for $(3 S, 9 R)$ one has 3 conformers above $10 \%$ population-based on $\Delta \mathrm{E}$-statistics, 2 conformers above $10 \%$ based on $\Delta \mathrm{G}$-statistics; 6 conformers between $10 \%$ and $5 \%$ population-based on $\Delta \mathrm{E}, 1$ conformer in the same range based on $\Delta \mathrm{G}$. For $(3 S, 9 S)$ one has 3 conformers above $10 \%$ population-based on $\Delta \mathrm{E}$, 1 conformer above $10 \%$ based on $\Delta \mathrm{G} ; 2$ conformers between $10 \%$ and $5 \%$ population-based on $\Delta \mathrm{E}, 2$ conformers in the same range based on $\Delta \mathrm{G}$. A sizeable number of conformers is found to have population factors smaller than $5 \%$. It is interesting to note that most of the preferred conformers from $\Delta \mathrm{E}$ appear kind-of-rolled up and compact due to the presence of intramolecular hydrogen bonds $(\mathrm{HB})$ between the two $\mathrm{OH}$ groups, while the preferred $\Delta \mathrm{G}$-conformers are more elongated, with conformers exhibiting HB having smaller population factors. We believe that in the latter case, the absence of $\mathrm{HB}$ assures a higher larger entropic contribution, thus minimizing $\Delta \mathrm{G}$. This provides us with a further criterion to prefer the $\Delta \mathrm{E}$-choice over the $\Delta \mathrm{G}$-choice, since from the IR spectra in the $\mathrm{OH}$ stretching region (Figure 3) we may appreciate that internal HB is quite present.

Coming finally to consider the ECD spectra, in Figure 6 we report, in strict analogy to what done for VCD in Figure 5, the comparison of calculated spectra for $(3 S, 9 S)$ and $(3 S, 9 R)$ choices with the experimental ECD spectra. We think that, while based on $\triangle \mathrm{G}$-averages, there is no preference of $(3 S, 9 S)$ over $(3 S, 9 R)$ (or just a slight preference of the latter over the former, due to the presence of a non-observed negative hump at a low wavelength in the calculated ECD spectrum of the former, based on $\triangle \mathrm{E}$ we should reject the $(3 S, 9 S)$-choice.

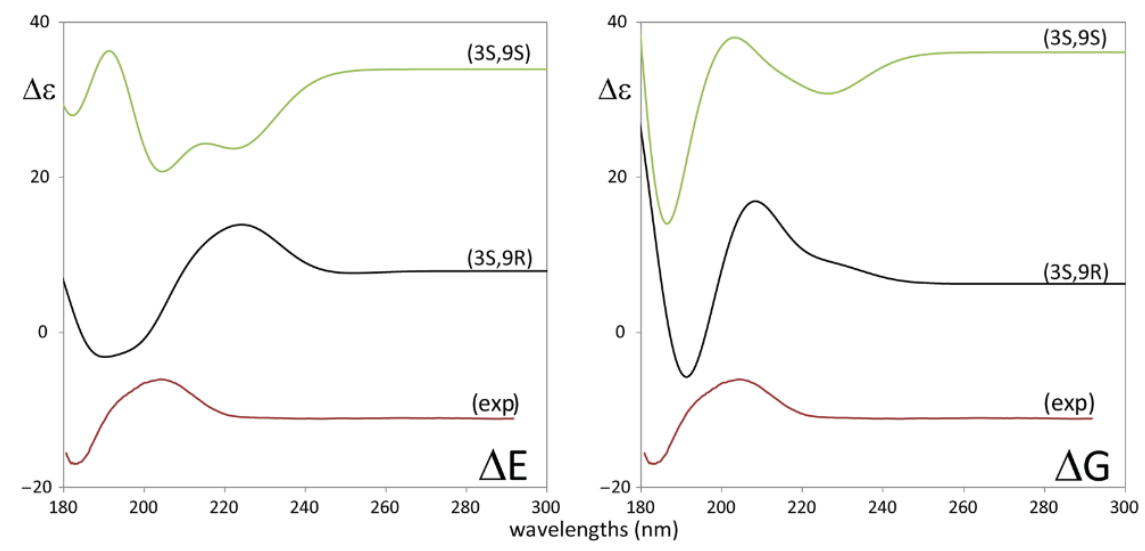

Figure 6. Comparison of experimental ECD (top) spectra of 9-hydroxynerolidol, (1), with corresponding TD-DFT calculated spectra of $(3 S, 9 R)-\mathbf{1}$ and $(3 S, 9 S)-\mathbf{1}$ with two different Boltzmann averages: on the left average carried out based on $\Delta \mathrm{E}$, on the right average carried out based on $\Delta \mathrm{G}$. No wavelength shift applied to calculated spectra. No shift was applied.

As a final comment on this "Results and Discussion" section, we notice that there is a contradiction between the VCD/ECD/DFT results and the Mosher's method NMR data about the configuration of stereogenic carbon 9 of sesquiterpene 1 . The former methods indicate a (slight) preference for $(R)$, the latter one for $(S)$. This is a bit of a problem and in the conclusions section, we'll list the facts in favor of either choice. Instead, the choice for stereogenic carbon atom 3 is definitely $(S)$, within the limits of the difficulty of the present 
problem, related to the huge number of conformers and to interactions with the solvent, which have not been treated in the present work (related to the latter aspect, we point out that VCD spectra were recorded in $\mathrm{CCl}_{4}$, an apolar, very symmetric solvent). Stereogenic carbon 3 is shared by all three compounds examined here and it is reassuring to state that, due to spectroscopic similarities, especially in the ECD spectra, the configuration of carbon 3 is the same in the three compounds and is $(S)$, a characteristic which appears robust and maintained in the members of the whole family. Moreover, we are glad to be in accord with the configuration of carbon 3 with the previous conclusions by Merten et al. (2017) on linear diterpenes extracted from algae [26,27].

\section{Conclusions}

In this work, we have investigated, by chiroptical spectroscopic methods, namely VCD and ECD allied to DFT calculations and ancillary theoretical-statistical analysis, the three related linear farnesane-type sesquiterpenes 9-hydroxynerolidol (1), 9-oxonerolidol (2), and chiliadenol B (3), isolated from C. lopadusanus, which is an endemic and native plant growing in the Lampedusa Island (Sicily, Italy). The three compounds have shown interesting activity against human pathogens, e.g., Gram+ and Gram- bacteria resistant to the antibiotic. We believe the AC assignment is beneficial to understand the action mechanism of potential drugs that could be based on these compounds. We have established, through VCD and ECD spectra allied with DFT calculations that the configuration of the stereogenic carbon atom 3 is (S). For compound 1 VCD and ECD show a slight preference for stereogenic carbon 9 to be in the $(R)$ configuration (vis-à-vis the similarity index parameters), while the application of the advanced Mosher method, which has recently found widespread use on natural products [18], indicates that the $(S)$ choice for the configuration of carbon atom 9 is preferable. This contradictory result should be viewed with some benevolence since the number of admissible conformers is between 20 and 50 , which makes the problem quite challenging for any technique.

Finally, we remark that the very same AC assignment for carbon 3 was made also for some linear diterpenes extracted from algae of the Mediterranean Sea, a fact which may not be so fortuitous, shedding some light onto the biochemistry of the two different organisms, one terrestrial and one marine, yet from the same geographic area, from which the different linear terpenes were extracted.

Supplementary Materials: The following are available online at https:/ /www.mdpi.com/article/ 10.3390/biom11121902/s1, Figure S1: graphical representations of SI as a function of scaling factors SF for the AC two choices $(3 S, 9 R)$ and $(3 S, 9 S)$; Figures S2-S4 for the 3D-representation of $(3 S, 9 R)$-conformers ordered in $\triangle E$-population factors; Figures S5-S7 for 3D-representation of (3S,9R)conformers ordered in $\Delta \mathrm{G}$-population factors; Figures S8-S11: the 3D-representation of $(3 S, 9 S)$ conformers ordered in $\triangle \mathrm{E}$-population factors. Figures S12-S14: 3D-representation of (3S,9S)-conformers ordered in $\triangle$ G-population factors, Table S1: Similarity Indices SI and Sim_NN for selected values of scaling factors for the four possible AC choices, $(3 S, 9 R),(3 S, 9 S),(3 S, 9 R),(3 S, 9 R)$; Table S2: energetics in $\Delta \mathrm{E}$ and $\Delta \mathrm{G}$ for $\mathrm{AC}(3 S, 9 R)$; Table S3: energetics in $\Delta \mathrm{E}$ and $\Delta \mathrm{G}$ for $\mathrm{AC}(3 S, 9 S)$.

Author Contributions: The Authors have a good record of amicable collaboration. The work has been designed in the group of Napoli (A.C., M.M. and A.E.), which is an expert in Natural Products extraction, purification, and chemistry. It has been carried out in Brescia (G.M., G.L. and S.A.) where the chiroptical spectra have been taken and interpreted. The manuscript has been written with equal contributions from both groups. All authors have read and agreed to the published version of the manuscript.

Funding: This research was partly funded by the Italian Ministry of University and Research, through a grant from the PRIN 2017 program, number 2017A4XRCA_003. We also acknowledge help from Big \& Open Data Innovation Laboratory (BODaI-Lab), the University of Brescia, granted by Fondazione Cariplo and Regione Lombardia.

Acknowledgments: The authors thank Fabio Giovanetti who collected the plant in Lampedusa Island, Italy. A. Evidente is associated with the Istituto di Chimica Biomolecolare del CNR, Italy. 
Conflicts of Interest: The authors declare no conflict of interest.

\section{References}

1. Turner, W.B.; Aldridge, D.C. Fungal Metabolites II; Academic Press: New York, NY, USA, 1983.

2. Osbourn, A.E.; Lanzotti, V. Plant-Derived Natural Products; Springer: Dordrecht, The Netherlands; Heidelberg, Germany ; London, UK; New York, NY, USA, 2009; pp. 361-384.

3. Masi, M.; Nocera, P.; Reveglia, P.; Cimmino, A.; Evidente, A. Fungal metabolites antagonists towards plant pests and human pathogens: Structure-activity relationship studies. Molecules 2018, 23, 834. [CrossRef]

4. Cimmino, A.; Nimis, P.L.; Masi, M.; De Gara, L.; van Otterlo, W.A.; Kiss, R.; Evidente, A.; Lefranc, F. Have lichenized fungi delivered promising anticancer small molecules? Phytochem. Rev. 2019, 18, 1-36. [CrossRef]

5. Evidente, A.; Abouzeid, A.M.; Andolfi, A.; Cimmino, A. Recent Achievements in the Bio-control of Orobanche Infesting Important Crops in the Mediterranean Basin. J. Agric. Sci. Technol. 2011, 1, 461-483.

6. Cimmino, A.; Masi, M.; Evidente, M.; Superchi, S.; Evidente, A. Fungal phytotoxins with potential herbicidal activity: Chemical and biological characterization. Nat. Prod. Rep. 2015, 32, 1629-1653. [CrossRef] [PubMed]

7. Newman, D.J.; Cragg, G.M. Natural products as sources of new drugs from 1981 to 2014. J. Nat. Prod. 2016, 79, 629-661. [CrossRef]

8. Evidente, A.; Kornienko, A.; Cimmino, A.; Andolfi, A.; Lefranc, F.; Mathieu, V.; Kiss, R. Fungal metabolites with anticancer activity. Nat. Prod. Rep. 2014, 31, 617-627. [CrossRef] [PubMed]

9. Masi, M.; Maddau, L.; Linaldeddu, B.T.; Scanu, B.; Evidente, A.; Cimmino, A. Bioactive metabolites from pathogenic and endophytic fungi of forest trees. Curr. Med. Chem. 2018, 25, 208-252. [CrossRef] [PubMed]

10. Roscetto, E.; Masi, M.; Esposito, M.; Di Lecce, R.; Delicato, A.; Maddau, L.; Evidente, A.; Catania, M.R. Anti-Biofilm Activity of the fungal phytotoxin sphaeropsidin A against clinical isolates of antibiotic-resistant bacteria. Toxins 2020, 12, 444. [CrossRef]

11. Zito, P.; Sajeva, M.; Scirica, E.; Bruno, M.; Rosselli, S.; Maggio, A.; Senatore, F. Essential oils of Chiliadenus lopadusanus (Asteraceae). Nat. Prod. Commun. 2013, 8, 1159-1162. [CrossRef]

12. Masi, M.; Roscetto, M.; Zatout, R.; Cimmino, A.; Catania, M.R.; Surico, G.; Evidente, A. Farnesane-type sesquiterpenoids from Chiladenus lopadusanus with antibiotic activity. Antibiotic 2021, 10, 148. [CrossRef]

13. Stoessl, A.; Stothers, J.B.; Ward, E.W.B. The structures of some stress metabolites from Solanum melongena. Can. J. Chem. 1975, 53, 3351-3358. [CrossRef]

14. Hiroi, M.; Takaoka, D. Non-volatile sesquiterpenoids in the leaves of camphor tree (Cinnamomum camphora). Nippon. Kagaku Kaishi 1974, 762-765. (In Japanese) [CrossRef]

15. Vlad, P.; Souček, M. On terpenes. CXXXVII. Absolute configuration of nerolidol. Collect. Czechoslov. Chem. Commun. 1962, 27, 1726-1729. [CrossRef]

16. Hegazy, M.E.F.; Matsusa, H.; Nakamura, S.; Hussein, T.A.; Yoshikawa, M.; Parè, P.W. Chemical constituents and their antibacterial and antifungal activity from the Egyptian herbal medicine. Chiliadenus Montanus Phytochem. 2014, 103, 154-161. [CrossRef] [PubMed]

17. Evidente, A.; Andolfi, A.; Cimmino, A. Relationships between the stereochemistry and biological activity of fungal phytotoxins. Chirality 2011, 23, 674-693. [CrossRef] [PubMed]

18. Evidente, A.; Cimmino, A.; Andolfi, A. The Effect of Stereochemistry on the Biological Activity of Natural Phytotoxins, Fungicides, Insecticides and Herbicides. Chirality 2013, 25, 59-78. [CrossRef] [PubMed]

19. Cimmino, A.; Masi, M.; Evidente, M.; Superchi, S.; Evidente, A. Application of Mosher's method for absolute configuration assignment to bioactive plants and fungi metabolites. J. Pharm. Biomed. Anal. 2017, 144, 59-89. [CrossRef] [PubMed]

20. He, Y.; Bo, Y.; Dukor, R.K.; Nafie, L.A. Determination of Absolute Configuration of Chiral Molecules Using Vibrational Optical Activity: A Review. Appl. Spectrosc. 2011, 65, 699-723. [CrossRef]

21. Keiderling, T.A. Structure of Condensed Phase Peptides: Insights from Vibrational Circular Dichroism and Raman Optical Activity Techniques. Chem. Rev. 2020, 120, 3381-3419. [CrossRef]

22. Polavarapu, P.L.; Santoro, E. Vibrational optical activity for structural characterization of natural products. Nat. Prod. Rep. 2020, 37, 1661-1699. [CrossRef]

23. Batista, J.M., Jr.; Blanch, E.W.; Bolzani, V.S. Recent advances in the use of vibrational chiroptical spectroscopic methods for stereochemical characterization of natural products. Nat. Prod. Rep. 2015, 32, 1280-1302. [CrossRef] [PubMed]

24. Del Rio, R.E.; Joseph-Nathan, P. Vibrational Circular Dichroism Absolute Configuration of Natural Products from 2015 to 2019. Nat. Prod. Commun. 2021, 16, 1-30. [CrossRef]

25. Abbate, S.; Burgi, L.F.; Castiglioni, E.; Lebon, F.; Longhi, G.; Toscano, E.; Caccamese, S. Assessment of configurational and conformational properties of Naringenin by Vibrational Circular Dichroism. Chirality 2009, 21, 436-441. [CrossRef]

26. Merten, C.; Smyrniotopoulos, V.; Tasdemir, D. Assignment of absolute configurations of highly flexible linear diterpenes from the brown alga Bifurcaria bifurcata by VCD spectroscopy. Chem. Commun. 2015, 51, 16217-16220. [CrossRef] [PubMed]

27. Smyrniotopoulos, V.; Merten, C.; Kaiser, M.; Tasdemir, D. Bifurcatriol, a New Antiprotozoal Acyclic Diterpene from the Brown Alga Bifurcaria bifurcate. Mar. Drugs 2017, 15, 245-255. [CrossRef] [PubMed]

28. Polavarapu, P.L.; Donahue, E.A.; Shanmugam, G.; Scalmani, G.; Hawkins, E.K.; Rizzo, C.; Ibnusaud, I.; Thomas, G.; Habel, D.; Sebastian, D. A Single Chiroptical Spectroscopic Method May Not Be Able To Establish the Absolute Configurations of Diastereomers: Dimethylesters of Hibiscus and Garcinia Acids. J. Phys. Chem. A 2011, 115, 5665-5673. [CrossRef] 
29. Frisch, M.J.; Trucks, G.W.; Schlegel, H.B.; Scuseria, G.E.; Robb, M.A.; Cheeseman, J.R.; Scalmani, G.; Barone, V.; Petersson, G.A.; Nakatsuji, H.; et al. Gaussian 16; Revision, C.01. Gaussian, Inc.: Wallingford, CT, USA, 2016.

30. Stephens, P.J. Theory of Vibrational Circular Dichroism. J. Phys. Chem. 1985, 89, 745-752. [CrossRef]

31. Fusè, M.; Mazzeo, G.; Longhi, G.; Abbate, S.; Masi, M.; Evidente, A.; Puzzarini, C.; Barone, V. Unbiased Determination of Absolute Configurations by vis-à-vis Comparison of Experimental and Simulated Spectra: The Challenging Case of Diplopyrone. J. Phys. Chem. B 2019, 123, 9230-9237. [CrossRef] [PubMed]

32. Paoloni, L.; Mazzeo, G.; Longhi, G.; Abbate, S.; Fusè, M.; Bloino, J.; Barone, V. Toward Fully Unsupervised Anharmonic Computations Complementing Experiment for Robust and Reliable Assignment and Interpretation of IR and VCD Spectra from Mid-IR to NIR: The Case of 2,3-Butanediol and trans-1,2-Cyclohexanediol. J. Phys. Chem. A 2020, 124, 1011-1024. [CrossRef]

33. Herzberg, G. Infrared and Raman Spectra of Polyatomic Molecules. In Molecular Spectra and Molecular Structure, 2nd ed.; David. Van Nostrand Co.: Toronto, ON, Canada; New York, NY, USA; London, UK, 1945.

34. Laux, L.; Pultz, V.M.; Abbate, S.; Havel, H.A.; Overend, J.; Moscowitz, A.; Lightner, D.A. Inherently dissimetric chromophores and Vibrational Circular Dichroism. The CH2-CH2-C*H Fragment. J. Am. Chem. Soc. 1982, 104, 4276-4278. [CrossRef]

35. Cao, X.; Shah, R.D.; Dukor, R.K.; Guo, C.; Freedman, T.B.; Nafie, L.A. Extension of Fourier transform vibrational circular dichroism into the near infrared region: Continuous spectral coverage from 800 to $10,000 \mathrm{~cm}^{-1}$. Appl. Spectrosc. 2004, 58, 1057-1064. [CrossRef] [PubMed]

36. Aviles Moreno, J.R.; Ureña Horno, E.; Partal Ureña, F.; López González, J.J. IR-Raman-VCD study of R-(+)-Pulegone: Influence of the solvent. Spectrochim. Acta A 2011, 79, 767-776. [CrossRef] [PubMed]

37. Hartwig, B.; Suhm, M.A. Subtle hydrogen bonds: Benchmarking with OH stretching fundamentals of vicinal diols in the gas phase. Phys. Chem. Chem. Phys. 2021, 23, 21623-21640. [CrossRef]

38. Moscowitz, A.; Mislow, K. Inherently Dissymmetric Chromophores: The Absolute Configuration of (-)-trans-Cyclooctene. J. Am. Chem. Soc. 1962, 84, 4605-4606. [CrossRef]

39. Beecham, A.F. The Influence of Allylic Oxygen on the $\pi \rightarrow \pi^{*}$ CD of Certain Chromophores. Tetrahedron 1971, $27,5207-5216$. [CrossRef]

40. Lightner, D.A.; Gurst, J.E. Chapter 12: Dienes. In Organic Conformational Analysis and Stereochemistry from Circular Dichroism Spectroscopy; Wiley-VCH: New York, NY, USA, 2000; Chapter 10.

41. Autschbach, J. Computing chiroptical properties with first-principles theoretical methods: Background and illustrative examples. Chirality 2009, 21, E116-E152. [CrossRef] [PubMed]

42. Covington, C.L.; Polavarapu, P.L. Similarity in Dissymmetry Factor Spectra: A Quantitative Measure of Comparison between Experimental and Predicted Vibrational Circular Dichroism. J. Phys. Chem. A 2013, 117, 3377-3386. [CrossRef]

43. Shen, J.; Zhu, C.; Reiling, S.; Vaz, R.A. Novel Computational Method for Comparing Vibrational Circular Dichroism Spectra. Spectrochim. Acta A 2010, 76, 418-422. [CrossRef] [PubMed]

44. Shen, J.; Li, Y.; Vaz, R.; Izumi, H. Revisiting Vibrational Circular Dichroism Spectra of (S)-(+)-Carvone and (1S,2R,5S)-(+)-Menthol Using SimIR/VCD Method. J. Chem. Theory Comput. 2012, 8, 2762-2768. [CrossRef] [PubMed]

45. Debie, E.; De Gussem, E.; Dukor, R.K.; Herrebout, W.; Nafie, L.A.; Bultinck, P.A. Confidence Level Algorithm for the Determination of Absolute Configuration Using Vibrational Circular Dichroism or Raman Optical Activity. Chem. Phys. Chem. 2011, 12, 1542-1549. [CrossRef]

46. Mazzeo, G.; Cimmino, A.; Masi, M.; Longhi, G.; Maddau, L.; Memo, M.; Evidente, A.; Abbate, S. Importance and Difficulties in the Use of Chiroptical Methods to Assign the Absolute Configuration of Natural Products: The Case of Phytotoxic Pyrones and Furanones produced by Diplodia corticola. J. Nat. Prod. 2017, 80, 2406-2415. [CrossRef] [PubMed] 